\title{
Epithelial ciliated beating cells essential for ex vivo ALI culture growth
}

\author{
Delphine Gras ${ }^{1 \dagger}$, Aurélie Petit ${ }^{2 \dagger}$, Jérémy Charriot², Lucie Knabe²,3 Khuder Alagha ${ }^{4}$, Anne Sophie Gamez ${ }^{2}$, \\ Céline Garulli', Arnaud Bourdin²,3, Pascal Chanez ${ }^{1,4}$, Nicolas Molinari, ${ }^{5,6}$ and Isabelle Vachier ${ }^{2,7^{*}}$ (i)
}

\begin{abstract}
Background: Bronchial epithelium plays a key role in orchestrating innate and adaptive immunity. The fate of ex vivo airway epithelial cultures growing at the air liquid interface (ALI) derived from human endobronchial biopsies or brushings is not easy to predict. Calibrating and differentiating these cells is a long and expensive process requiring rigorous expertise. Pinpointing factors associated with ALI culture success would help researchers gain further insight into epithelial progenitor behavior.
\end{abstract}

Methods: A successful ALI culture was defined as one in which a pseudostratified epithelium has formed after 28 days in the presence of all differentiated epithelial cell types. A 4-year prospective bi-center study was conducted with adult subjects enrolled in different approved research protocols.

Results: 463 consecutive endobronchial biopsies were obtained from normal healthy volunteers, healthy smokers, asthmatic patients and smokers with COPD. All demographic variables, the different fiber optic centers and culture operators, numbers of endo-bronchial biopsies and the presence of ciliated cells were carefully recorded. Univariate and multivariate models were developed. A stepwise procedure was used to select the final logistic regression model. ALI culture success was independently associated with the presence of living ciliated cells within the initial biopsy $(\mathrm{OR}=2.18[1.50-3.16], p<0.001)$.

Conclusion: This finding highlights the properties of the cells derived from the epithelium dedifferentiation process. The preferential selection of samples with ciliated beating cells would probably save time and money. It is still unknown whether successful ALI culture is related to indicators of general cell viability or a purported stem cell state specifically associated with ciliated beating cells.

\section{Background}

Bronchial epithelial cells represent the primary airway defense barrier against inhaled pathogens and particles $[1,2]$. This epithelium plays a crucial role in maintaining airway homeostasis by regulating innate and acquired immunity [3] through the production of a wide range of cytokines, chemokines and mediators. The bronchial epithelium involves a pseudostratified organization of basal, ciliated, goblet and other less common types of cells $[1,4]$. These different cell types appear to be essential for proper function $[1,2]$.

\footnotetext{
* Correspondence: isabelle.vachier@inserm.fr

${ }^{\dagger}$ Equal contributors

${ }^{2}$ Department of Respiratory Diseases, CHRU Montpellier, Montpellier, France ${ }^{7} \mathrm{CHU}$ Montpellier, Hôpital Arnaud de Villeneuve, 371 Av Doyen G Giraud, 34295 Montpellier Cx 5, France

Full list of author information is available at the end of the article
}

The majority of initial studies compared epithelial content in experiments performed on biopsy, lung resection or autopsy specimens, but only recently on bronchoscopy specimens [5]. Epithelial cell culture techniques have been well documented for many years, including studies on cell lines (immortalized or not) and on primary cells obtained from volunteers who submitted to brushings [6] or forceps biopsies [7] (used as primary cells or after monolayer cultures). Studies with cell lines are easy, reproducible and not very expensive, whereas those carried out with primary cultures are hampered by the high cost, between-donor variability, the dissociation process, as well as the limited number of cells suitable for experiments. In monolayer cultures, the number of passages is critical and cells clearly undergo a dedifferentiation process and lose their phenotype [8]. This has led to the development of new cell-line systems involving a 
monolayer culture followed by an air-liquid interface (ALI) using an appropriate medium to preserve cell phenotypes $[9,10]$. This human bronchial epithelial cell (HBEC) model can generate polarized pseudostratified epithelium composed of most known cell types [11]. This culture system provides a useful tool for the in vitro study of airway epithelial biological features and cell differentiation $[11,12]$, including functional studies of specific differentiated cells (i.e. ciliary beating, mucus secretion and cytokine production).

Implementation of the ALI technique is currently the focus of an increasing number of publications because it reproduces a well differentiated airway epithelium with distinct, functional cells [13]. Many factors may contribute to the success or failure of ALI cultures, including initial sample types and amounts, underlying disease, sample handling, techniques and media $[14,15]$.

In this study, we aimed to identify potential predictive factors for obtaining a successful ALI culture that could be used in future experiments. We investigated these variables in all experiments performed in our laboratory on bronchial biopsies obtained from healthy volunteers, smokers, asthmatic (mild, moderate, and severe) subjects and COPD patients.

\section{Methods}

\section{Subjects}

Four hundred sixty-three adults were consecutively included from March 2009 to July 2013 at the Hôpital Arnaud de Villeneuve (Montpellier, France) and the Assistance Publique des Hôpitaux de Marseille (Marseille, France). They all signed an informed consent form in order to participate in a study approved by the ethics committees of our institutions for the purpose of a specific biomedical research project on epithelial pathophysiologic mechanisms.

Controls $(n=83)$ and smokers $(n=65)$ without COPD (no asthma, no allergies, normal chest X-ray and pulmonary function tests) underwent bronchoscopy for various purposes (foreign body removal, suspected but unconfirmed hemoptysis or peripheral nodules $(<10$ $\mathrm{mm}$ ) found during CT scan examination) and had normal macroscopic airway appearance. Smokers were defined by a smoking status greater than 10 pack-years.

Asthma diagnosis $(n=175)$ was based on the assessment of clinical features consistent with asthma and evidence of variable expiratory airflow obstruction during functional testing $\left(\mathrm{FEV}_{1}\right.$ increase of at least $12 \%$ and 200 $\mathrm{ml}$ after inhalation of $200 \mu \mathrm{g}$ of salbutamol or after 4 weeks of oral corticosteroid treatment, an average daily diurnal PEF variability $<10 \%)$. Asthma severity was evaluated according to the current global initiative for asthma guidelines (http://ginasthma.org/). Patients with severe asthma $(n=124)$ also met the American Thoracic
Society criteria for refractory asthma [16]. Asthmatic patients had been free of respiratory infections and asthma exacerbations for at least 6 weeks at the time of inclusion. They were current non-smokers or had a smoking history of less than 5 pack-years [17].

COPD diagnosis $(n=130)$ was based on evidence of non-reversible obstructive airflow $\mathrm{FEV}_{1} / \mathrm{FVC}<0.7, \mathrm{FEV}_{1}$ improvement after inhaling 200 to $400 \mu \mathrm{g}$ of albuterol below $12 \%$ and $200 \mathrm{ml}$, and a smoking history greater than 10 pack-years without any evidence of any alternative diagnosis according to the best standards of care (i.e. no history of any other respiratory disease based on clinical examination or computed tomography scan findings) [18].

\section{Endobronchial biopsy}

Flexible bronchoscopies were usually performed under local anesthesia and two biopsies from each donor were removed using alligator forceps (Olympus) on a subsegmental bronchus of the left lower lobe, as previously described [19]. Briefly, following premedication with subcutaneous atropine $(0.25 \mathrm{mg})$ and midazolam $(5 \mathrm{mg})$, local naso-pharyngeal anesthesia was implemented using lidocaine $(1-5 \%$, with a maximum individual dose of $300 \mathrm{mg}$ ). Then a fiber optic bronchoscope (Olympus BF20) was nasally inserted into the trachea.

\section{Cell culture}

Primary human bronchial epithelial cells were obtained from bronchial biopsy specimens and cultured under ALI conditions, as previously described [14]. Briefly, bronchial epithelial biopsy tissue was dissociated and suspended in bronchial epithelial growth medium (Lonza). After an expansion phase in monolayers, cells were plated on uncoated nucleopore membranes (24$\mathrm{mm}$ dia., $0.4-\mathrm{mm}$ pore size, Transwell Clear) in a 1:1 mixture of bronchial epithelial growth medium and Dulbecco's modified Eagle's medium (Lonza) applied only at the basal side to establish the ALI. Cells were cultured for 28 days to obtain a polarized and differentiated cell population with a mucociliary phenotype.

\section{Immunofluorescence}

ALI cultured cells were fixed in situ on inserts and transferred to glass slides for analysis. Cells were fixed using $10 \%$ formalin and blocked/permeabilized with PBS, $10 \%$ donkey serum, $1 \%$ BSA, and $0.1 \%$ Triton-X. Cells were incubated with appropriate primary antibodies at $4^{\circ} \mathrm{C}$ overnight (mouse monoclonal anti- $\beta$-tubulin IV (ONS.1A6, Sigma-Aldrich), mouse monoclonal anti-Muc5AC (45M1, Abcam) and goat polyclonal anti-p63 (S16, Santa Cruz Biotechnology)). Alexa Fluor ${ }^{\circ} 488$, Alexa Fluor 555 or Alexa Fluor 647 secondary labelling (Invitrogen) was applied for $1 \mathrm{~h}$ at room temperature and incubated with 
DAPI before mounting. Negative controls were incubated with secondary labelling antibodies only. Cells were visualized under a Zeiss axioimager microscope using axiovision software (Zeiss).

\section{Data format and statistical analysis}

We defined three successful culture steps. First, we considered that a monolayer culture was successful when cells were growing in monolayers but did not reach the ALI transfer stage (denoted "ML"). Then we considered that the ALI passage was successful when cells were able to reach the ALI step without a successful 28-day culture (denoted"pALI"). Finally, ALI culture was considered successful when a pseudostratified epithelium was obtained with the presence of at least basal, ciliated and goblet cells and without any contamination after 28 days of ALI culture (denoted "ALI 28d").

Continuous parametric data are presented as means \pm standard deviation (SD), and categorical variables as numbers and percentages. We used the $\chi^{2}$ test for categorical variables. A multivariate analysis was conducted using a Cox regression model to determine independent factors of success, in which we included all variables associated with a $p$-value of below 0.20 in the univariate analysis. Then a stepwise procedure allowed us to obtain the final multivariate model. Survival curves were estimated with the Kaplan Meier method and the logrank test was used. Statistical tests were performed using R (version 3.1.0 (2014-04-10) R Foundation for Statistical Computing) software. The significance level was set at $p \leq 0.05$.

\section{Results}

\section{Patient characteristics}

Table 1 summarizes the demographic and clinical characteristics of subjects included in the study.

As expected, $\mathrm{FEV}_{1}$ was significantly decreased in severe asthma and COPD patients, while $\mathrm{FEV}_{1} / \mathrm{FVC}$ was decreased in COPD. Moreover, treatment intake is more important in severe asthma and COPD than in mildmoderate asthmatics and controls or smokers.

Biopsy cultures from asthmatic patients were mainly performed and obtained at the Marseille (MRS) center, while those for COPD and smokers were mainly from Montpellier (MTP).

\section{HBEC cultures}

Overall, $65 \%$ of biopsy cultures were successfully grown as monolayers in flasks and no differences in success were noted between groups of subjects. The major reason for unsuccessful monolayer culture was bacterial and/or fungal contamination at an early stage. In such cases, the cultures were excluded in order to avoid any further contamination within the incubator. The other finding at this stage was the incapacity of cells to adhere and grow. In such cases, cells were left in culture for up to 2 weeks and were then stopped.

Thereafter, $47 \%$ of samples were transferred into transwells for the first liquid-liquid interface step. Finally $40 \%$ of the total initial biopsy specimens were successful in ALI culture at 28 days and could be used for further experiments (Fig. 1a). At this stage, a well differentiated pseudostratified epithelium was obtained with the presence of at least basal, ciliated and goblet cells (Fig. 1b).

Table 1 Demographic and clinical characteristics of subjects

\begin{tabular}{|c|c|c|c|c|c|c|}
\hline & $\begin{array}{l}\text { Overall } \\
N=463\end{array}$ & $\begin{array}{l}\text { Control } \\
N=83\end{array}$ & $\begin{array}{l}\text { Smokers } \\
N=65\end{array}$ & $\begin{array}{l}\text { COPD } \\
N=130\end{array}$ & $\begin{array}{l}\text { Mild-Mod A } \\
N=61\end{array}$ & $\begin{array}{l}\text { Severe } A \\
N=124\end{array}$ \\
\hline $\operatorname{Sex} F(n, \%)$ & $214(47)$ & $49(60)$ & $20(31)$ & $22(17)$ & $39(66)$ & $84(68)$ \\
\hline Age $($ mean $\pm s d)$ & $54.7 \pm 14.4$ & $50.1 \pm 17.0$ & $53.5 \pm 14.2$ & $62.4 \pm 10.2$ & $52.5 \pm 14.6$ & $51.5 \pm 13.1$ \\
\hline BMl & $24.3 \pm 4.7$ & $23.5 \pm 4.6$ & $24.9 \pm 4.8$ & $23.8 \pm 4.2$ & $22.4 \pm 2.8$ & $27.9 \pm 6.3$ \\
\hline $\mathrm{FEV}_{1} \%$ & $78.8 \pm 22.7$ & $97.8 \pm 12.9$ & $96.4 \pm 18.4$ & $64.2 \pm 20.5$ & $89.6 \pm 15.0$ & $71.3 \pm 19.1$ \\
\hline FVC \% & $94.8 \pm 21.7$ & $98.2 \pm 19.4$ & $103.5 \pm 21.9$ & $88.7 \pm 20.8$ & $112.4 \pm 15.4$ & $83.5 \pm 14.5$ \\
\hline FEV1/FVC & $64.8 \pm 14.0$ & $78.5 \pm 9.5$ & $75.6 \pm 6.0$ & $55.7 \pm 11.1$ & $69.8 \pm 10.7$ & $59.1 \pm 13.7$ \\
\hline ICS (n, \%) & $172(37)$ & $0(0)$ & $0(0)$ & $33(25)$ & $38(62)$ & $101(81)$ \\
\hline OCS (n, \%) & $70(15)$ & $6(7)$ & $4(6)$ & $4(3)$ & $2(3)$ & $54(44)$ \\
\hline $\operatorname{SABA}(n, \%)$ & $102(22)$ & $0(0)$ & $0(0)$ & $15(12)$ & $15(25)$ & $71(57)$ \\
\hline LABA $(n, \%)$ & $165(36)$ & $0(0)$ & $0(0)$ & $36(28)$ & $28(46)$ & $99(80)$ \\
\hline LAMA (n, \%) & $23(5)$ & $0(0)$ & $0(0)$ & $18(24)$ & $1(2)$ & $3(2)$ \\
\hline MTP/MRS & $204 / 259$ & $34 / 49$ & $64 / 1$ & $82 / 48$ & $9 / 52$ & 15/109 \\
\hline $\begin{array}{l}\text { Biopsy Number } \\
(\text { mean } \pm s d)\end{array}$ & $1.57 \pm 1.09$ & $1.58 \pm 1.16$ & $2.88 \pm 0.96$ & $1.90 \pm 1.28$ & $1.25 \pm 0.85$ & $1.07 \pm 0.40$ \\
\hline Biopsies grown by a senior operator $(n, \%)$ & $259(70)$ & $48(77)$ & $32(49)$ & $69(53)$ & $37(79)$ & $73(85)$ \\
\hline
\end{tabular}




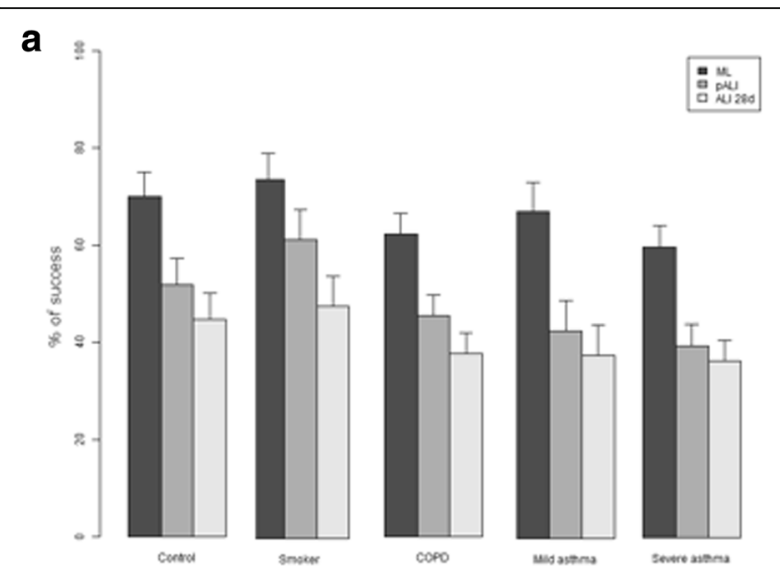

b
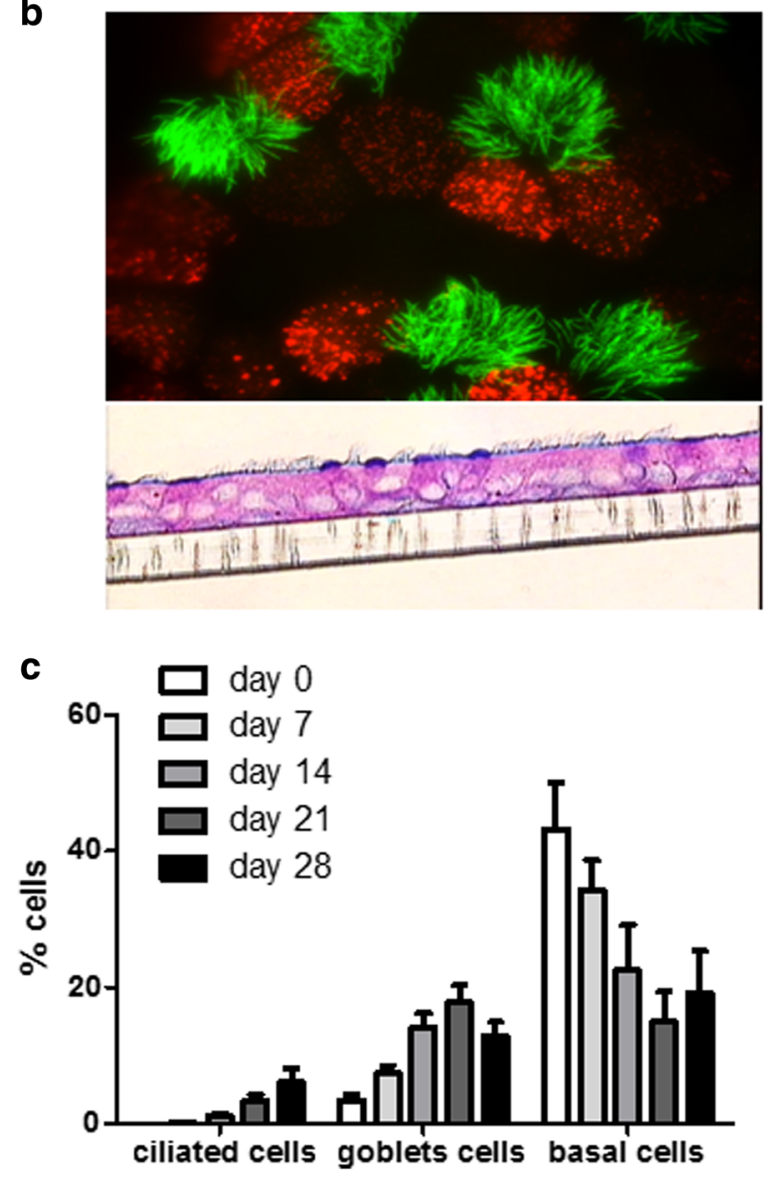

Fig. 1 a Percentage of success in obtaining a monolayer culture $(\mathrm{ML})$, the filter passage (pALI) and complete success at 28 days of ALI culture (ALI 28d) in controls, smokers, COPD, mild and severe asthma samples. $\mathbf{b}$ A representative figure of an ALI culture at 28 days differentiation. c Percentage of epithelial cells (ciliated, goblet and basal cells) during the differentiation process at 28 days ALI culture using immunofluorescence with specific antibody against $\beta$-tubulin IV, Muc5AC and p63, respectively
The percentage of ciliated and goblet cells increased from day 0 to day 28 , whereas the percentage of basal cells did not change or tended to decrease (Fig. 1c).

\section{Factors associated with ALI culture at $\mathbf{2 8}$ days}

Univariate analysis indicated that 28-day ALI culture success was associated with the absence of inhaled corticosteroid use $(\mathrm{HR}=0.64[0.45-0.92] ; p=0.02)$, long acting $\beta$ agonist use $(\mathrm{HR}=0.62[0.43-0.88] ; p=0.01)$, and the presence of beating ciliated cells within the initial bronchial biopsy ( $\mathrm{HR}=2.18[1.50-3.16] ; p<0.001)$. Univariate analysis results are tabulated in the supplemental data file (see Additional file 1).

According to multivariate analysis, the success in obtaining a useful 28-day ALI culture was associated only with the presence of beating ciliated cells (supplemental online cell videos obtained from biopsy samples are provided; see Additional files 2 and 3).

Success with and without the presence of beating ciliated cells in the initial bronchial biopsy

We obtained a significantly higher percentage of success in generating a well pseudostratified epithelium in 28day ALI cultures for all pathology conditions ( $p=$ $0.00001,0.000002$ and 0.0002 according to the $\chi^{2}$ test for ML, pALI and ALI 28d, respectively), when beating ciliated cells were present at the beginning of the process, just after the bronchoscopy procedure (Fig. 2).

In order to test the effects of the presence of beating ciliated cells in the initial biopsy, we applied a Cox proportional hazards model (Fig. 3). Clearly, the presence of beating ciliated cells in the initial biopsy is associated with the success rate.

\section{Discussion}

In the present study, we aimed to describe our expertise in the development of human bronchial epithelial cell ALI cultures obtained from human bronchial biopsies, and to identify potential predictive factors associated with the success of this process after 28 days of culture. We consecutively included the 463 bronchial biopsies taken in order to develop ALI cultures at out two specialized centers in control subjects as well as in pathological conditions. We drew up an exhaustive list of patient characteristics and procedure details which could interfere with the success rate. The main finding of the multivariate analysis was that the presence of ciliated beating epithelial cells at the beginning of the procedure, just after the bronchial biopsy specimen was obtained, was the only parameter associated with the percentage of success in obtaining a well pseudostratified epithelium in a 28-day ALI culture.

Bronchial epithelium is characterized by the presence of differentiated epithelial cell types organized as a 


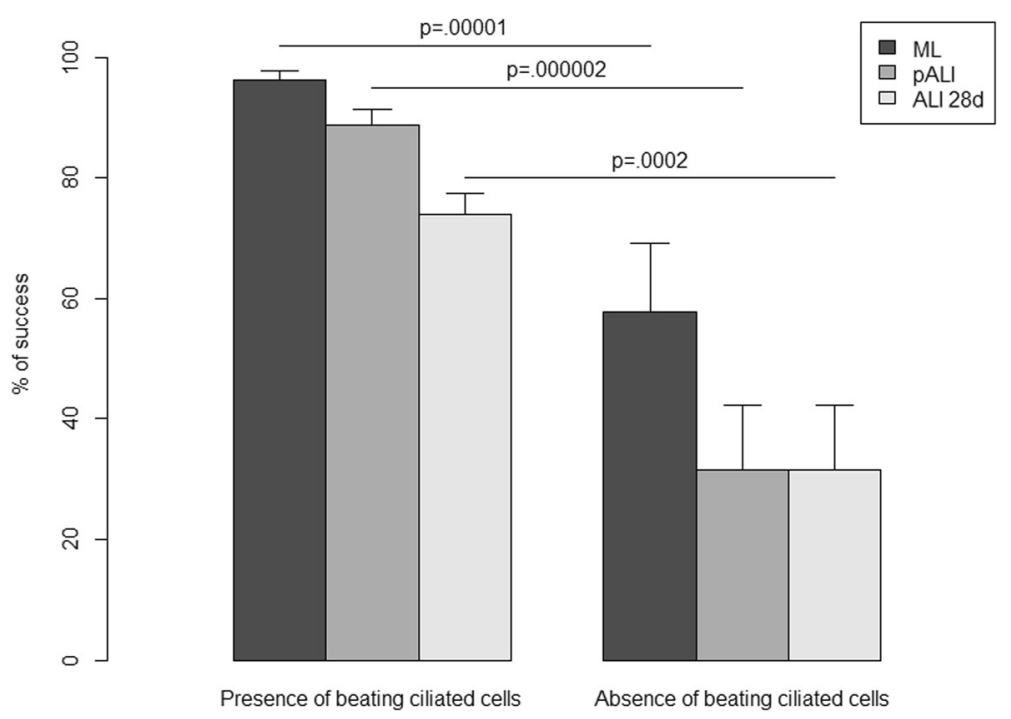

Fig. 2 Percentage of success in obtaining a monolayer culture (ML), the filter passage (pALI) and complete success at 28 days of ALI culture (ALI 28d) in bronchial biopsies from all patients significantly differed between cultures obtained from initial biopsies where beating ciliated cells were present versus not $\left(p=.00001, .000002\right.$ and .0002 , respectively, according to a $\chi^{2}$ test)

pseudostratified epithelium. Currently, and since the beginning of in vitro studies on human bronchial epithelial cells, cultures come mainly from the sources described hereafter [8]. Carcinoma-derived cells formed confluent polarized monolayers but lacked pseudostratification and the presence of ciliated cells, even though this effect might be counteracted when cultured in ALI [20]. Virus transformed cell lines form polarized multilayer cultures but often lack mucus production and relevant genes might be altered or not expressed [21]. Finally, primary

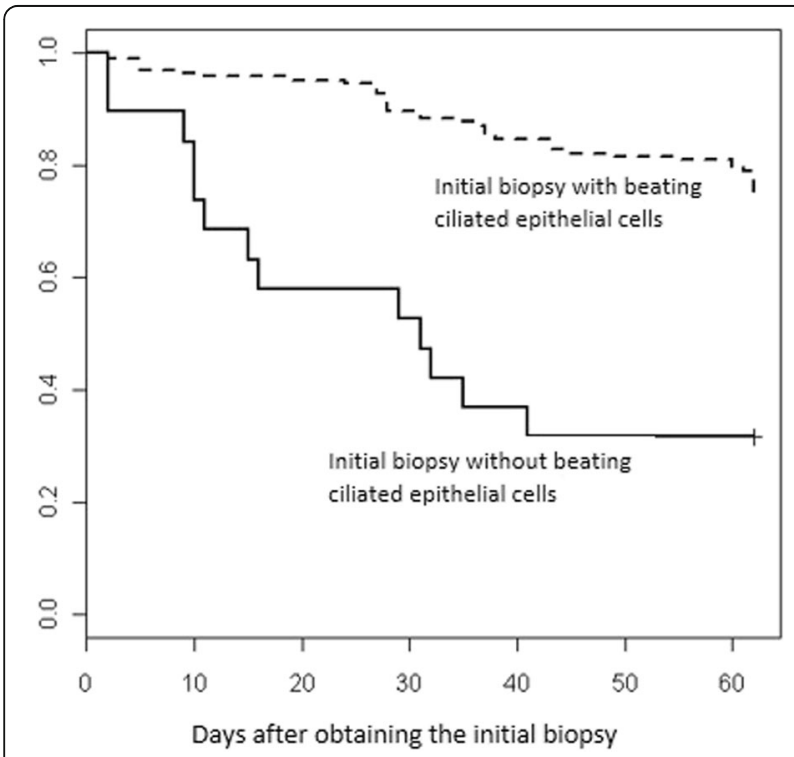

Fig. 3 Cox model applied to assess the time of success for cultures differentiated with the presence or not of beating ciliated epithelial cells cells were found to result in well differentiated cultures representative of a human bronchial phenotype but with a limited lifespan. Culturing at an ALI is essential for polarity and differentiation in these cells [11].

For 10 years now, we have thus focused on finding technologies that result in well pseudostratified structures as well as other in vivo-like differentiation characteristics. Primary bronchial epithelial cells specifically showed a mucociliary differentiation pattern comparable to that noted in vivo [22]. This technique for obtaining in vitro epithelium is now widely used, but the model has yet to be clearly characterized, even though the persistence of the pathological phenotype has been well described [14, 15]. The generation of differentiated cultures of normal human bronchial epithelial cells is a time- and labour-intensive procedure, especially when producing a large number of cell cultures for analysis [23]. This is why we wanted to describe optimal conditions for reliably obtaining epithelial cultures for further experiments, and to address a list of factors which could predict this success. Our study does not address expected growth patterns for bronchial brushing samples, which is another source of cells already in use [6]. This study was performed on all consecutive samples obtained in our two specialized centres from 2009 to 2013. The results revealed that only $40 \%$ of the samples were successfully cultured, which is a low rate. However, the latter reflects the real world difficulties associated with training and practice. In follows that all predictive conditions which might further increase yield are being sought. 
In order to better describe the dynamic changes that occur during the culture of differentiated epithelia, we were able-in a subset of culture wells from different subject phenotypes-to count the number of ciliated, goblet and basal cells by using specific labelled antibodies. In the initial mucociliary differentiation step (day 0 of ALI culture), we detected a population of basal epithelial stem cells $\left(\mathrm{p} 63^{+}\right)$and undifferentiated cells, which then decreased over time. It has been reported that basal stem/progenitor cells are involved in the regeneration of human bronchial epithelium [24]. Basal and undifferentiated epithelial cells decreased during mucociliary differentiation, whereas goblet and ciliated cells were found to increase from day 7 to day 14, respectively, to reach maximal differentiation at 28 days. These findings are in accordance with previous studies describing the mucociliary differentiation of normal human tracheobronchial cells [25].

Our major finding was the partial explanation for 28day culture success as being due to the presence, in the original biopsy, of ciliated beating cells. One of the limitations of our study was that we did not assess the histological features of the initial biopsy. We chose instead to use all of the initial material obtained from the bronchoscopy in order to increase culture success. It is still unclear whether this result reflects the true presence of ciliated cells, which may act as progenitors, or the fact that visual beating is an index of better viability of the original cells. Under physiological conditions, normal adult human bronchial epithelium turnover is relatively slow, i.e. approximately every 1 to 4 months [26], and the basal cells are relatively quiescent. in vivo lineagetracing studies in pseudostratified mucociliary epithelium from mouse trachea have shown that basal cells can function as classical stem cells and both self-renew and give rise to ciliated and secretory cells [27]. Notch signalling promotes this differentiation, favouring the production of ciliated cells and high levels of secretory cell fate [28]. Therefore, differentiated cells are commonly thought to send back signals to their respective stem and progenitor cells to regulate their proliferation and differentiation [29]. The term dedifferentiation was used to suggest that differentiated epithelial cells revert to a previous developmental stage before their subsequent differentiation into an alternative cell fate [30]. These authors highlighted the existence of multiple cellular reservoirs of regenerative capacity, which may enable a more effective reparative response.

Moreover, light microscopy observations readily revealed the presence of beating cilia when the biopsy was viewed just before cell detachment at the outset of monolayer culturing. The verification of cell viability could easily relate to better cell behaviour leading to increased cell adherence for further dedifferentiation/differentiation processes.
The biology and relevance of bronchial epithelium stem cells remain unclear. Identification of the cellular and molecular mechanisms of bronchial stem cells involved in repair, proliferation, and mucociliary differentiation under normal and pathological conditions will provide further insight into cellular differentiation with regard to the development of new therapeutic strategies or airway reconstruction methods for obtaining, for instance, artificial trachea.

\section{Conclusion}

This is the first study to characterize the properties of cells derived from the bronchial epithelium dedifferentiation process. The presence of ciliated beating epithelial cells was the only parameter associated with success in obtaining a well pseudostratified epithelium in a 28-day ALI culture. The latter finding will influence our practice towards the preferential selection of samples when ciliated beating cells in order to save time and money.

\section{Additional files}

Additional file 1: Table with the univariate analysis results. (DOCX $14 \mathrm{~kb}$ )

Additional file 2: Video S1. Showing cells obtained from the biopsy sample with the presence of beating ciliated cells. (AVI 1483 kb)

Additional file 3: Video S2. showing cells obtained from the biopsy sample with the presence of beating ciliated cells. (AVI $785 \mathrm{~kb}$ )

\section{Abbreviations \\ ALI 28d: Air liquid interface at 28 days; ALI: Air liquid interface; BMl: Body mass index; COPD: Chronic obstructive pulmonary Disease; CT scan: Computer tomography; FEV1: Forced expiratory volume in 1 second; FVC: Forced vital capacity; HBEC: Human bronchial epithelial cell; HR: Hazard ratio; ML: Monolayer; MRS: Marseille; MTP: Montpellier; pALI: Air liquid interface passage; PEF: Pulmonary expiratory function}

\section{Acknowledgements}

The authors would to thank all the bronchoscopists and the patients for making our work possible.

\section{Funding}

This study was supported by grants from the French Agence Nationale de Recherche (ANR) Mucocil project (ANR-13BSV5-0015-02), the Programme Hospitalier de Recherche Clinique (PHRC IR) AP-HM (2013-14), and CHRU Montpellier (AOI 9244).

\section{Availability of data and material}

The datasets generated during the current study are not publicly available due to confidentiality concerns, but are available from the corresponding author and statistics department upon (reasonable) request.

\section{Authors' contributions}

$A B, P C$, IV made substantial contributions to the conception and design of the study; DG, AP, JC, LK, KA, ASG, CG were responsible for experiments and data acquisition; NM performed analyses and AB, PC, DG, AP, IV were involved in data interpretation; IV, NM wrote the manuscript; all authors read, improved and gave final approval for the manuscript.

\section{Competing interest}

The authors declare that they have no competing interests.

Consent for publication

Not applicable. 


\section{Ethics approval and consent to participate}

All patients signed an informed consent form in order to participate in a study approved by the ethics committees at our institutions for the purpose of a specific biomedical research project on epithelial pathophysiologic mechanisms. They were included in 3 different studies reported as followed: Programme Hospitalier de Recherche Clinique (PHRC IR) AP-HM (2013-14 - NCT01947218) with approval from the Sud Méditérranée II ethics committee; CHRU Montpellier (AOI 9244 - NCT02354677) with approval from the Sud Méditérranée III ethics committee; COBRA Project from the Inserm (2008 A00294-51) with approval from the lle de France / ethics committee.

\section{Publisher's Note}

Springer Nature remains neutral with regard to jurisdictional claims in published maps and institutional affiliations.

\section{Author details \\ ${ }^{1}$ UMR INSERM U1067 CNRS 7333, Aix Marseille University, Marseille, France. 2Department of Respiratory Diseases, CHRU Montpellier, Montpellier, France. ${ }^{3}$ U1046 INSERM, UMR9214 CNRS, Montpellier University, Montpellier, France. ${ }^{4}$ Department of Respiratory Medicine, Assistance Publique Hopitaux de Marseille, Aix Marseille University, Marseille, France. ${ }^{5}$ Institut Montpelliérain Alexander Grothendieck, CNRS, UM, Montpellier, France. ${ }^{6}$ Department of Statistics, CHRU Montpellier, Montpellier, France. ${ }^{7} \mathrm{CHU}$ Montpellier, Hôpital Arnaud de Villeneuve, 371 Av Doyen G Giraud, 34295 Montpellier Cx 5, France.}

Received: 7 October 2016 Accepted: 26 April 2017

Published online: 03 May 2017

\section{References}

1. Davies DE. Epithelial Barrier Function and Immunity in Asthma. Ann Am Thorac Soc. 2014;11(Supplement 5):S244-51.

2. Whitsett JA, Alenghat T. Respiratory epithelial cells orchestrate pulmonary innate immunity. Nat Immunol. 2015;16(1):27-35.

3. Schleimer RP, Kato A, Kern R, Kuperman D, Avila PC. Epithelium: at the interface of innate and adaptive immune responses. J Allergy Clin Immunol. 2007;120(6):1279-84.

4. Knight DA, Holgate ST. The airway epithelium: structural and functional properties in health and disease. Respirol Carlton Vic. 2003:8(4):432-46.

5. National Institutes of Health workshop summary. Summary and recommendations of a workshop on the investigative use of fiberoptic bronchoscopy and bronchoalveolar lavage in individuals with asthma. J Allergy Clin Immunol. 1985;76(2 Pt 1):145-7.

6. Stokes AB, Kieninger E, Schögler A, Kopf BS, Casaulta C, Geiser T, et al. Comparison of three different brushing techniques to isolate and culture primary nasal epithelial cells from human subjects. Exp Lung Res. 2014;40(7): 327-32

7. Ramirez RD, Sheridan S, Girard L, Sato M, Kim Y, Pollack J, et al. Immortalization of human bronchial epithelial cells in the absence of viral oncoproteins. Cancer Res. 2004;64(24):9027-34.

8. Prytherch Z, Job C, Marshall H, Oreffo V, Foster M, BéruBé K. Tissue-Specific stem cell differentiation in an in vitro airway model. Macromol Biosci. 2011; 11(11):1467-77.

9. Yamaya M, Finkbeiner WE, Chun SY, Widdicombe JH. Differentiated structure and function of cultures from human tracheal epithelium. Am J Physiol Lung Cell Mol Physiol. 1992;262(6):L713-24.

10. Whitcutt MJ, Adler KB, Wu R. A biphasic chamber system for maintaining polarity of differentiation of cultured respiratory tract epithelial cells. Vitro Cell Dev Biol J Tissue Cult Assoc. 1988;24(5):420-8.

11. Wu R, Sato GH, Whitcutt MJ. Developing differentiated epithelial cell cultures: airway epithelial cells. Fundam Appl Toxicol Off J Soc Toxicol. 1986. 6(4):580-90

12. Pezzulo AA, Starner TD, Scheetz TE, Traver GL, Tilley AE, Harvey B-G, et al. The air-liquid interface and use of primary cell cultures are important to recapitulate the transcriptional profile of in vivo airway epithelia. Am J Physiol Lung Cell Mol Physiol. 2011;300(1):L25-31.

13. Sellgren KL, Butala EJ, Gilmour BP, Randell SH, Grego S. A biomimetic multicellular model of the airways using primary human cells. Lab Chip. 2014;14(17):3349-58
14. Gras D, Bourdin A, Vachier I, de Senneville L, Bonnans C, Chanez P. An ex vivo model of severe asthma using reconstituted human bronchial epithelium. J Allergy Clin Immunol. 2012;129(5):1259-66.e1.

15. Gamez AS, Gras D, Petit A, Knabe L, Molinari N, Vachier I, et al. Supplementing defect in club cell secretory protein attenuates airway inflammation in COPD. Chest. 2015;147(6):1467-76.

16. Chanez P, Wenzel SE, Anderson GP, Anto JM, Bel EH, Boulet L-P, et al. Severe asthma in adults: what are the important questions? J Allergy Clin Immunol. 2007;119(6):1337-48.

17. Reddy AP, Gupta MR. Management of asthma: the current US and European guidelines. Adv Exp Med Biol. 2014;795:81-103.

18. Cooper CB, Barjaktarevic I. A new algorithm for the management of COPD. Lancet Respir Med. 2015;3(4):266-8.

19. Vachier I, Chiappara G, Vignola AM, Gagliardo R, Altieri E, Térouanne B, et al. Glucocorticoid receptors in bronchial epithelial cells in asthma. Am J Respir Crit Care Med. 1998;158(3):963-70.

20. Grainger Cl, Greenwell LL, Lockley DJ, Martin GP, Forbes B. Culture of Calu-3 cells at the air interface provides a representative model of the airway epithelial barrier. Pharm Res. 2006;23(7):1482-90.

21. Forbes B, Ehrhardt C. Human respiratory epithelial cell culture for drug delivery applications. Eur J Pharm Biopharm Off J Arbeitsgemeinschaft Für Pharm Verfahrenstechnik EV. 2005 Jul;60(2):193-205

22. Fulcher ML, Gabriel S, Burns KA, Yankaskas JR, Randell SH. Well-differentiated human airway epithelial cell cultures. Methods Mol Med. 2005;107:183-206.

23. Aufderheide M, Förster C, Beschay M, Branscheid D, Emura M. A new computer-controlled air-liquid interface cultivation system for the generation of differentiated cell cultures of the airway epithelium. Exp Toxicol Pathol Off J Ges Für Toxikol Pathol. 2016 Jan;68(1):77-87

24. Stripp BR, Reynolds SD. Maintenance and repair of the bronchiolar epithelium. Proc Am Thorac Soc. 2008;5(3):328-33.

25. Ross AJ, Dailey LA, Brighton LE, Devlin RB. Transcriptional profiling of mucociliary differentiation in human airway epithelial cells. Am J Respir Cell Mol Biol. 2007:37(2):169-85.

26. Breeze RG, Wheeldon EB. The cells of the pulmonary airways. Am Rev Respir Dis. 1977;116(4):705-77.

27. Pardo-Saganta A, Tata PR, Law BM, Saez B, Chow RD-W, Prabhu M, et al. Parent stem cells can serve as niches for their daughter cells. Nature. 2015; 523(7562):597-601.

28. Rock JR, Gao X, Xue Y, Randell SH, Kong Y-Y, Hogan BLM. Notch-dependent differentiation of adult airway basal stem cells. Cell Stem Cell. 2011;8(6):639-48.

29. Tadokoro T, Gao X, Hong CC, Hotten D, Hogan BLM. BMP signaling and cellular dynamics during regeneration of airway epithelium from basal progenitors. Dev Camb Engl. 2016 Mar 1;143(5):764-73

30. Tata PR, Mou H, Pardo-Saganta A, Zhao R, Prabhu M, Law BM, et al. Dedifferentiation of committed epithelial cells into stem cells in vivo. Nature. 2013;503(7475):218-23.

\section{Submit your next manuscript to BioMed Central and we will help you at every step:}

- We accept pre-submission inquiries

- Our selector tool helps you to find the most relevant journal

- We provide round the clock customer support

- Convenient online submission

- Thorough peer review

- Inclusion in PubMed and all major indexing services

- Maximum visibility for your research

Submit your manuscript at www.biomedcentral.com/submit 